\title{
Clinique juridique des droits fondamentaux
}

Mémoire pour le Conseil constitutionnel dans le cadre de l'examen du projet de loi relatif à l'immigration, à l'intégration et à la nationalité, 18 mai 2011 [extraits]

\section{Xavier Aurey}

\section{OpenEdition}

\section{Journals}

Édition électronique

URL : https://journals.openedition.org/crdf/5425

DOI : $10.4000 /$ crdf. 5425

ISSN : 2264-1246

Éditeur

Presses universitaires de Caen

Édition imprimée

Date de publication : 1 décembre 2011

Pagination : 85-90

ISBN : 978-2-84133-396-7

ISSN : 1634-8842

Référence électronique

Xavier Aurey, "Clinique juridique des droits fondamentaux », Cahiers de la recherche sur les droits fondamentaux [En ligne], 9 | 2011, mis en ligne le 01 décembre 2012, consulté le 15 novembre 2022. URL : http://journals.openedition.org/crdf/5425; DOI : https://doi.org/10.4000/crdf.5425 


\title{
Clinique juridique des droits fondamentaux ${ }^{1}$
}

\section{Mémoire pour le Conseil constitutionnel dans le cadre de l'examen du projet de loi relatif à l'immigration, à l'intégration et à la nationalité, 18 mai 2011 [extraits] ${ }^{2}$}

\author{
Xavier AUREY (dir.) \\ Doctorant à I'Université Panthéon-Assas - Paris II \\ Chercheur associé au Centre de recherche sur les droits fondamentaux et les évolutions du droit (CRDFED)
}

V. L'inconstitutionnalité des zones d'attente ad hoc

A. La remise en cause de garanties légales d'exigences constitutionnelles

B. L'erreur manifeste d'appréciation dans la définition de circonstances à même de justifier une restriction des droits et libertés et une différence de traitement

VI. La question du maintien simultané d'un nombre important d'étrangers en zone d'attente

VII. L'application de l'article 11 aux zones d'attente ad hoc

VIII. L'effectivité du contrôle du juge sur le maintien en zone d'attente

A. Le contrôle par le juge des irrégularités

B. L'étendue du pouvoir du juge et l'effectivité de son contrôle

\section{L'inconstitutionnalité des zones d'attente ad hoc}

Article 10 alinéa 2: «Après le premier alinéa de l'article L. 221-2 du même code, il est inséré un alinéa ainsi rédigé: “Lorsqu'il est manifeste qu'un groupe d'au moins dix étrangers vient d'arriver en France en dehors d'un point de passage frontalier, en un même lieu ou sur un ensemble de lieux distants d'au plus dix kilomètres, la zone d'attente s'étend, pour une durée maximale de vingt-six jours, du ou des lieux de découverte des intéressés jusqu'au point de passage frontalier le plus proche"».

1. La Clinique juridique des droits fondamentaux, créée en 2009 au sein du CRDFED, a pour vocation de réunir enseignants, chercheurs, étudiants (et praticiens éventuellement), et de permettre la formation à la recherche par l'étude de cas concrets. Voir http://www.unicaen.fr/recherche/ $\mathrm{mrsh} / \mathrm{crdfed} / \mathrm{clinique}$

2. Le texte intégral de ce mémoire (réalisé sous la direction de Marie-Joëlle Redor-Fichot) est disponible sur http://www.scribd.com/doc/55796794/ CJDF-memoire-mai-2011. Ont participé à la rédaction de ce mémoire [en italique les membres de l'équipe ayant contribué directement à la rédaction de l'extrait] : membres enseignants: Marie-Joëlle Redor-Fichot (directrice de la Clinique), Grégory Godiveau (maître de conférences, directeur adjoint de la Clinique), Xavier Aurey (doctorant, secrétaire général de la Clinique), Samuel Etoa (maitre de conférences), Nathalie Havas (docteur en droit), Juliette Lecame (doctorante au CRDFED). Membres étudiants (Master 2 Contentieux des droits fondamentaux) : Émilie AïtMehdi, Alice Lukacs, Jennifer Lelouey, Maya Oberlin, Ghofran Ounissi, Andréa Pagnucco et Chloé Viel. 
Créée en 1992 sous le nom de zone de transit, la «zone d'attente» correspond légalement au lieu qui «s'étend des points d'embarquement et de débarquement à ceux où sont effectués les contrôles des personnes. Elle peut inclure, sur l'emprise, ou à proximité, de la gare, du port ou de l'aéroport ou à proximité du lieu de débarquement, un ou plusieurs lieux d'hébergement assurant aux étrangers concernés des prestations de type hôtelier. Dans ces lieux d'hébergement, un espace permettant aux avocats de s'entretenir confidentiellement avec les étrangers est prévu $»^{3}$. La création d'une telle zone répond à la nécessité de gérer l'arrivée sur le territoire français d'étrangers n'étant pas autorisés à y entrer ou qui demandent leur admission au titre de l'asile ${ }^{4}$. Votre Conseil a toujours analysé la mesure de placement en zone d'attente comme une restriction de la liberté d'aller et venir de l'étranger affectant sa liberté individuelle au sens de l'article 66, mais n'entraînant pas à son encontre un degré de contrainte comparable à celui qui résulterait de son placement en centre de rétention ${ }^{5}$.

Actuellement, les étrangers qui se voient refuser l'entrée sur le territoire français ne peuvent être placés que dans des zones d'attente créées de manière durable et spécialement aménagées pour l'accueil du public sur plusieurs jours (les autorités peuvent ainsi, en cas de besoin, étendre une zone d'attente à tout lieu d'hébergement pouvant offrir aux étrangers des "prestations de type hôtelier») et pour l'accueil spécifique d'étrangers ( «un espace permettant aux avocats de s'entretenir confidentiellement avec les étrangers est prévu ${ }^{6}$ ).

\section{A. La remise en cause de garanties légales d'exigences constitutionnelles}

Par définition créées en dehors de toute considération quant à l'existence d'une structure d'accueil pour les étrangers, les zones d'attente ad hoc entraînent le risque certain de faire résider les étrangers en cause dans des structures non adaptées à leur hébergement et ne répondant pas ainsi au minimum de garanties nécessaires au respect de la dignité humaine dont vous avez affirmé la valeur constitutionnelle ${ }^{7}$. Par essence temporaires et créées en dehors de toute structure adaptée, ces zones d'attente ad hoc font également courir le risque certain de voir une reconnaissance affaiblie des droits normalement garantis aux étrangers en zone d'attente. Ainsi, les services d'assistance, de consultation d'un médecin, d'un interprète ou d'un avocat sont rendus d'autant plus difficiles que la zone d'attente ainsi créée sera plus ou moins accessible (zone de montagne, bord de mer isolé...). Fondées sur les principes de respect de la dignité de la personne humaine et de droit à un recours effectif, ces garanties légales ne peuvent qu'être regardées comme le standard minimum applicable aux étrangers en zone d'attente. Priver, même temporairement, les étrangers de tels droits et en amoindrir la portée compte tenu des conditions de création et d'accès à ces zones d'attente constitue donc une atteinte disproportionnée au texte constitutionnel.

Nous vous demandons ainsi de censurer l'article 10 de cette loi en tant qu'il prive de garanties légales les exigences constitutionnelles liées au respect de la dignité de la personne humaine et au droit à un recours effectif.

En elles-mêmes problématiques, ces restrictions prennent une ampleur particulière dans le cas de demandeurs d'asile ou de mineurs isolés, des catégories de personnes nécessitant une protection accrue.

Concernant les demandeurs d'asile, vous avez pu rappeler «qu'un étranger qui a sollicité son admission en France au titre de l'asile ne saurait faire l'objet d'un maintien en zone de transit le temps nécessaire à son départ, moyennant des garanties adéquates, que s'il apparaît que sa demande d'asile est manifestement infondée » ${ }^{8}$. Vous avez de plus souligné qu' "Aux termes du quatrième alinéa du préambule de la Constitution de 1946: “Tout homme persécuté en raison de son action en faveur de la liberté a droit d'asile sur les territoires de la République". Il incombe au législateur d'assurer en toutes circonstances l'ensemble des garanties légales que comporte cette exigence constitutionnelle ${ }^{9}$.

Concernant les mineurs isolés, l'article L. 221-5 du CESEDA prévoit que «Lorsqu'un étranger mineur non accompagné d'un représentant légal n'est pas autorisé à entrer en France, le procureur de la République, avisé immédiatement par l'autorité administrative, lui désigne sans délai un administrateur ad hoc. Celui-ci assiste le

3. Code de l'entrée et du séjour des étrangers et du droit d'asile (CESEDA), art. L. 221-2.

4. CESEDA, art. L. 221-1.

5. CC, 25 février 1992, déc. $n^{\circ}$ 92-307 DC, Loi portant modification de l'ordonnance $n^{\circ}$ 45-2658 du 2 novembre 1945 modifiée relative aux conditions d'entrée et de séjour des étrangers en France, cons. 14 et 15: Le maintien en zone de transit «n'entraîne pas à l'encontre de l'intéressé un degré de contrainte sur sa personne comparable à celui qui résulterait de son placement dans un centre de rétention en application de l'article 35 bis de l'ordonnance précitée. 15. Mais considérant que le maintien d'un étranger en zone de transit, en raison de l'effet conjugué du degré de contrainte qu'il revêt et de sa durée, a néanmoins pour conséquence d'affecter la liberté individuelle de la personne qui en fait l'objet au sens de l'article 66 de la Constitution; que si la compétence pour décider du maintien peut être confiée par la loi à l'autorité administrative, le législateur doit prévoir, selon des modalités appropriées, l'intervention de l'autorité judiciaire pour que celle-ci exerce la responsabilité et le pouvoir de contrôle qui lui reviennent».

6. CESEDA, art. L. 221-2.

7. CC, 27 juillet 1994, déc. $n^{\circ}$ 94-343/344 DC, Loi relative au respect du corps humain et loi relative au don et à l'utilisation des éléments et produits du corps humain, à l'assistance médicale à la procréation et au diagnostic prénatal, cons. 2.

8. CC, 25 février 1992, déc. $n^{\circ}$ 92-307 DC, Loi portant modification de l'ordonnance $n^{\circ}$ 45-2658 du 2 novembre 1945 modifiée relative aux conditions d'entrée et de séjour des étrangers en France, cons. 9 à 11.

9. CC, 22 avril 1997, déc. $\mathrm{n}^{\circ}$ 97-389 DC, Loi portant diverses dispositions relatives à l'immigration, cons. 25 . 
mineur durant son maintien en zone d'attente et assure sa représentation dans le cadre des procédures administratives et juridictionnelles relatives à ce maintien ». Face à une situation de maintien en zone d'attente en ellemême difficile pour les mineurs isolés, cette garantie légale constitue le standard minimum acceptable pour leur protection. Or, il est manifeste que dans le cadre d'une zone d'attente ad hoc, l'accès à une telle assistance sera rendu plus difficile pour le mineur isolé, d'autant que la loi ici étudiée ne prévoit aucun système spécifique de garantie.

Au regard des menaces spéciales qu'elles font peser sur les droits garantis aux demandeurs d'asile et aux mineurs isolés, et en l'absence de toute mesure spécifique concernant ces catégories de personnes,nous demandons au Conseil constitutionnel de juger l'article 10 de cette loi contraire à la Constitution en tant qu'il prive de garanties légales les exigences constitutionnelles de droit d'asile et de protection des mineurs isolés.

\section{B. L'erreur manifeste d'appréciation dans la définition de circonstances à même de justifier une restriction des droits et libertés et une différence de traitement}

Votre Conseil affirme depuis longtemps que le principe d'égalité «ne s'oppose pas à ce que le législateur déroge à l'égalité pour des raisons d'intérêt général dès lors que les différences de traitement qui en résultent sont en rapport avec l'objet de la loi qui les établit ${ }^{10}$. Le Comité des Droits de l'homme des Nations unies ${ }^{11}$ et la Cour européenne des Droits de l'homme ${ }^{12}$ ont une interprétation similaire de ce principe et considèrent qu'il n'y a pas rupture d'égalité lorsque la différence de traitement poursuit un but légitime dans une société démocratique et qu'elle respecte un rapport raisonnable de proportionnalité entre les moyens employés et le but visé. En l'espèce, seule une justification objective et raisonnable peut donc permettre de traiter différemment des étrangers pourtant placés dans une situation analogue.

Les étrangers susceptibles d'être placés en zone d'attente ad hoc sont en effet dans une situation analogue à celle des étrangers placés en zone d'attente traditionnelle au regard du refus d'entrée dont ils font l'objet. De plus, la création de ces zones d'attente ne peut qu'entraîner, comme démontré à notre point précédent, une privation temporaire des droits des étrangers et des restrictions supplémentaires apportées à l'exercice de ces droits, compte tenu du lieu possible de création de ces zones (zone de montagne, bord de mer isolé...).

Une telle différence de traitement ne pourra ainsi être jugée constitutionnelle que si elle répond à une justification objective et raisonnable au regard de l'objet de la loi. En ce sens, les travaux préparatoires à ce projet de loi nous montrent que l'argument principal quant à la création de telles zones réside dans le caractère exceptionnel et temporaire de la situation à laquelle elles doivent répondre.

Ainsi, comme l'a souligné François-Noël Buffet, rapporteur pour la Commission des lois du Sénat lors de l'examen de ce projet (19 janvier 2011), «Le but est de faire face à des situations exceptionnelles, tel le débarquement d'une centaine de Kurdes sur une plage de Corse du Sud en janvier 2010». En des termes proches, M. Thierry Mariani, rapporteur, énonce que «la possibilité de créer des zones d'attente temporaires devait être effectivement strictement limitée aux situations de débarquement d'un nombre important de migrants en dehors de points de passage frontalier ${ }^{13}$. M. Mariani continue en affirmant que «Cette exception au principe selon lequel les étrangers en situation irrégulière déjà présents sur le territoire relèvent des procédures d'éloignement et non des procédures de refus d'entrée ne peut en effet se justifier que dans des circonstances exceptionnelles, ne permettant pas aux autorités administratives de mettre en œuvre les procédures d'éloignement de droit commun. En effet, en cas d'arrivée simultanée de plusieurs dizaines de migrants en situation irrégulière, les procédures de non-admission et de placement en zone d'attente sont plus adaptées à la situation et doivent pouvoir être mises en œuvre ${ }^{14}$. M. Brice Hortefeux, lors de son audition par la Commission des lois du Sénat (21 décembre 2010), employait lui aussi des termes similaires: «Des événements récents ont montré que nous ne pouvons pas faire face à l'arrivée à la frontière d'un groupe important d'immigrants hors des points de passage répertoriés: je vous rappelle les perturbations causées en janvier dernier par le débarquement de 123 Kurdes sur une plage de Corse du Sud. Nous voulons donc donner un cadre juridique à ces situations exceptionnelles».

Tel que modifié par les parlementaires, l'article 10 de la loi soumise à votre examen prévoit que la création de telles zones d'attente ad hoc ne pourra avoir lieu que «Lorsqu'il est manifeste qu'un groupe d'au moins dix étrangers vient d'arriver en France en dehors d'un point de passage frontalier ». Il convient de savoir si l'arrivée d'un tel groupe de 10 personnes constitue une situation exceptionnelle,

10. Cf. CC, 13 janvier 1994, déc. n 93-331 DC, Loi rétablissant le renouvellement triennal par moitié des conseils généraux, cons. 10.

11. Cf. notamment le Comité des Droits de l'homme, 9 avril 1987, S.W.M. Broeks c. Pays-Bas, CCPR/C/29/D/172/1984

12. Cf. notamment $\mathrm{CEDH}, 23$ juillet 1968, Affaire "relative à certains aspects du régime linguistique de l'enseignement en Belgique» c. Belgique, $\mathrm{n}^{\circ} 1474 / 62$ et al.

13. Thierry Mariani, Rapport fait au nom de la Commission des lois constitutionnelles, de la législation et de l'administration générale de la République sur le projet de loi $\left(n^{\circ} 2400\right)$ relatif à l'immigration, à l'intégration et à la nationalité, Rapport $\mathrm{n}^{\circ} 2814$ enregistré à la présidence de l'Assemblée nationale le 16 septembre 2010, $\$$ II-B-1.

14. Ibid. 
seul motif d'intérêt général susceptible de justifier ici une dérogation au principe d'égalité.

Si l'article 61 de la Constitution ne confère pas à votre Conseil « un pouvoir général d'appréciation et de décision de même nature que celui du Parlement [...], il incombe au Conseil constitutionnel de s'assurer de l'absence de disproportion manifeste ${ }^{15}$ entre la mesure en cause et l'intérêt public protégé. Selon le rapport du 24 juin 2009 de la mission d'information sur les centres de rétention administrative et les zones d'attente, l'année 2008 a vu le placement en zone d'attente de 16645 personnes ${ }^{16}$, soit un peu plus de 45 personnes par jour. Même si la plupart des cas sont concentrés dans les zones des aéroports ParisCharles de Gaulle et Paris-Orly, ainsi que dans celle du Canet à Marseille, toutes ces zones ont les moyens d'accueillir plus d'une dizaine de personnes. De plus, les deux cas « exceptionnels» mentionnés par les débats parlementaires à titre d'exemple concernent le débarquement de 910 Kurdes sur une plage du Var en 2001 et de 123 Kurdes en Corse du Sud le 22 janvier 2010, des chiffres bien supérieurs à celui envisagé comme seuil par cet article.

Au regard de ces éléments, votre Conseil ne pourra que conclure que la seule condition de la présence d'un "groupe d'au moins dix étrangers [...] en un même lieu ou sur un ensemble de lieux distants d'au plus dix kilomètres » est loin de caractériser une situation comme exceptionnelle. À ce titre, le seuil de 10 personnes retenu par le législateur constitue une erreur manifeste d'appréciation de ce que peut être une situation exceptionnelle justifiant de déroger au principe d'égalité par le durcissement des conditions de maintien en zone d'attente (au titre de l'article 10 de cette loi).

Nous vous demandons ainsi de censurer l'article 10 de cette loi comme contraire à l'article 6 de la Déclaration des Droits de l'homme et du citoyen.

\section{La question du maintien simultané d'un nombre important d'étrangers en zone d'attente}

Article 11: "Après le premier alinéa de l'article L. 221-4 du Code de l'entrée et du séjour des étrangers et du droit d'asile, il est inséré un alinéa ainsi rédigé: "En cas de maintien simultané en zone d'attente d'un nombre important d'étrangers, la notification des droits mentionnés au premier alinéa s'effectue dans les meilleurs délais, compte tenu du nombre d'agents de l'autorité administrative et d'in- terprètes disponibles. De même, dans ces mêmes circonstances particulières, les droits notifiés s'exercent dans les meilleurs délais" ».

Tout individu qui voit sa liberté individuelle mise en cause doit pouvoir être «informé aussi rapidement que possible des droits qu'il peut exercer $"{ }^{17}$. L'article L. 221-4 du Code de l'entrée et du séjour des étrangers et du droit d'asile prévoit ainsi pour l'étranger placé en zone d'attente le droit de demander l'assistance d'un interprète et d'un médecin, ainsi que le droit de communiquer avec un conseil ou toute personne de son choix, et le droit de quitter la zone d'attente pour une destination hors de France. Ces droits sont autant de garanties du respect de la santé de l'étranger, de sa dignité, de sa liberté d'aller et venir et de sa sûreté, telles que protégées par la Constitution de 1958, le préambule de la Constitution de 1946 et la Déclaration des Droits de l'homme et du citoyen.

Cet article 11 prévoit une cause possible d'allongement du délai pendant lequel l'étranger ne sera pas informé des droits dont il peut bénéficier lors de son placement en zone d'attente. En temps normal, la Cour de cassation exige que la notification ne soit pas tardive et même qu'elle soit «imminente» ${ }^{18}$. Cette exigence est justifiée par le fait que tant que l'étranger ne s'est pas vu notifier les droits dont il est titulaire, il ne peut en bénéficier et se trouve donc dans une situation de quasi-vide juridique. Votre Conseil a pu juger également que «les dispositions prévoyant que cette notification est effectuée "dans les meilleurs délais" prescrivent une information qui, si elle ne peut être immédiate pour des raisons objectives, doit s'effectuer dans le plus bref délai possible» ${ }^{19}$. Une telle raison objective correspond à une raison étrangère au comportement de l'acteur qui souhaite s'en prévaloir. Il est donc nécessaire de voir si la combinaison d'un «nombre important d'étrangers » avec le «nombre d'agents de l'autorité administrative et d'interprètes disponibles » peut être qualifiée de «raison objective» autorisant un retard dans la notification des droits.

Pour pouvoir être qualifié de raison objective, ce «nombre important d'étrangers» ne doit pas correspondre à une situation normale ou proche de la normale pour la zone d'attente en cause. Le manque d'agents et d'interprètes serait alors le fait de l'État et ne pourrait donc justifier objectivement une telle limitation dans la notification de leurs droits aux étrangers.

En ce sens, le flou laissé autour de cette condition de nombre donne un pouvoir d'appréciation discrétionnaire à l'administration, ne permettant pas de distinguer de manière objective entre une situation normale et une

15. Notamment CC, 22 octobre 2009, déc. $\mathrm{n}^{\circ}$ 2009-590 DC, Loi relative à la protection pénale de la propriété littéraire et artistique sur Internet, cons. 18 à 20.

16. Mission d'information sur les centres de rétention administrative et les zones d'attente, Rapport d'information déposé en application de l'article 145 du Règlement par la Commission des lois constitutionnelles, de la législation et de l'administration générale de la République sur les centres de rétention administrative et les zones d'attente, Assemblée nationale, rapport nº 1776, 24 juin 2009, p. 12.

17. CC, 20 novembre 2003 , déc. $\mathrm{n}^{\circ} 2003-484$ DC, Loi relative à la maîtrise de l'immigration, au séjour des étrangers en France et à la nationalité, cons. 51.

18. Cass., $2^{\mathrm{e}}$ civ., 11 janvier $2001, \mathrm{n}^{\circ}$ 00-50.006.

19. CC, 20 novembre 2003, déc. $\mathrm{n}^{\circ} 2003-484$ DC, Loi relative à la maîtrise de l'immigration, au séjour des étrangers en France et à la nationalité, cons. 51 . 
situation pouvant nécessiter des mesures spéciales. Une suspension des droits de l'étranger pendant une certaine période étant en jeu, le mécanisme ne peut reposer sur une notion aussi imprécise, propre à faciliter l'arbitraire. En matière de protection de la liberté individuelle, vous exigez ainsi que toute loi soit accessible, précise et prévisible. Ainsi, vous avez condamné des dispositions dont «la formulation générale et imprécise [...] porte atteinte aux principes essentiels sur lesquels repose la protection de la liberté individuelle $»^{20}$. Votre interprétation est en ce sens commune à celle de la Cour européenne des Droits de l'homme qui, appelée à se prononcer en 1996 sur les zones d'attente en France, a pu juger qu' « une loi nationale autorisant une privation de liberté [... doit être] suffisamment accessible et précise afin d'éviter tout danger d'arbitraire ${ }^{21}$. Dans un sens très proche, la Cour de justice de l'Union européenne a de son côté jugé qu'une législation nationale conférant une compétence aux autorités de police doit, pour satisfaire l'exigence de sécurité juridique, "prévoir l'encadrement nécessaire de la compétence conférée à ces autorités afin, notamment, de guider le pouvoir d'appréciation dont disposent ces dernières dans l'application pratique de ladite compétence ${ }^{22}$.

Ainsi, l'article 11, tel que rédigé, contrevient aux exigences d'accessibilité et d'intelligibilité de la loi. En ce sens, il contrevient également à l'exigence d'une raison objective propre à justifier l'allongement limité du délai de notification de leurs droits aux étrangers, portant ainsi une atteinte disproportionnée à leur droit à la sûreté. Nous vous demandons donc de juger l'article 11 de cette loi contraire à l'objectif constitutionnel d'accessibilité et d'intelligibilité de la loi.

Article 45: «Les meilleurs délais au sens du deuxième alinéa s'entendent compte tenu du temps requis pour informer chaque étranger de ses droits lorsqu'un nombre important d'étrangers doivent être simultanément placés en rétention ».

Nous vous recommandons de vous référer à l'argumentation concernant l'article 11. En ce sens, nous vous demandons de juger l'article 45 non conforme à la Constitution.

\section{L'application de l'article 11 aux zones d'attente ad hoc}

Il est demandé au Conseil de reconnaître que les atteintes aux droits et libertés de l'étranger prennent une ampleur particulière lorsque les articles 10 et 11 de la loi sont combinés.
En effet, les zones d'attente ad hoc, pouvant par principe apparaître en tout lieu sur le territoire de la République, le personnel administratif nécessaire et les traducteurs ne seront pas immédiatement disponibles sur place. De ce fait, la notification puis l'exercice des droits reconnus à l'étranger seront toujours et automatiquement différés dans ces zones d'attente, comme le permet l'article 11. Ainsi, un étranger maintenu dans ces zones d'attente verra à chaque fois non seulement sa liberté d'aller et venir restreinte, mais également tous les autres droits dont il peut normalement se prévaloir. Si la conciliation des éléments de valeur constitutionnelle doit pencher en faveur de la sauvegarde de l'ordre public en ce qui concerne le maintien d'un étranger en zone d'attente, le cumul inévitable des atteintes aux différents droits et libertés de l'étranger dans les zones d'attente ad hoc doit rester exceptionnel. Or, l'application cumulée des articles 10 et 11 de la loi est inévitable. En cela, elle porte atteinte à la fois au droit à la sûreté, à la liberté d'aller et venir, au droit au respect de sa dignité humaine à un degré tel qu'il est ici impératif que la loi prévoie des conditions strictes pour la création de ces zones d'attente.

La loi ne prévoit aucune condition ou garantie pouvant contrer la généralisation de ces atteintes. Le degré de contrainte qu'une telle généralisation entraîne doit conduire à considérer que de telles zones d'attente relèvent de l'article 66 de la Constitution. Au regard de l'absence des garanties prévues par l'article 66 de la Constitution, nous vous demandons de censurer l'article 10 de cette loi comme contraire à la Constitution.

\section{L'effectivité du contrôle du juge sur le maintien en zone d'attente}

\section{A. Le contrôle par le juge des irrégularités}

Article 12: «À peine d'irrecevabilité, prononcée d'office, aucune irrégularité antérieure à l'audience relative à la première prolongation du maintien en zone d'attente ne peut être soulevée lors de l'audience relative à la seconde prolongation».

Aux termes de l'article 16 de la Déclaration des Droits de l'homme et du citoyen : «Toute Société dans laquelle la garantie des droits n'est pas assurée ni la séparation des pouvoirs déterminée, n’a point de Constitution». Vous avez reconnu «que sont garantis par cette disposition le droit des personnes intéressées à exercer un recours juridictionnel effectif, le droit à un procès équitable ${ }^{23}$. En ce sens, comme le souligne à juste titre la Cour européenne des Droits de l'homme, «le droit à un procès équitable ne peut

20. CC, 18 janvier 1995, déc. $n^{\circ}$ 94-352 DC, Loi d'orientation et de programmation relative à la sécurité, cons. 18.

21. CEDH, 25 juin 1996, Amuur c. France, nº 17/1995/523/609, \$ 50.

22. CJUE, 22 juin 2010, Aziz Melki et Sélim Abdeli, C-188/10 et C-189/10.

23. CC, 27 juillet 2006, déc. $\mathrm{n}^{\circ}$ 2006-540 DC, Loi relative au droit d'auteur et aux droits voisins dans la société de l'information, cons. 11 ; voir également CC, 9 avril 1996, déc. n 96-373 DC, Loi organique portant statut d'autonomie de la Polynésie française, cons. 83. 
passer pour effectif que si les demandes et les observations des parties sont vraiment “entendues", c'est-à-dire dûment examinées par le tribunal saisi. Autrement dit, l'article 6 implique à la charge du "tribunal" l'obligation de se livrer à un examen effectif des moyens, arguments et offres de preuve des parties, sauf à en apprécier la pertinence ${ }^{24}$.

Selon l'article 12 du projet de loi, aucune irrégularité antérieure à la première audience décidant de la prolongation en zone d'attente ne peut être soulevée lors de l'audience relative à la seconde prolongation. Or, dans certains cas, établir une liste exhaustive des irrégularités survenues avant la première audience se révèle une tâche impossible, faute de moyens, ou de temps nécessaire à l'appréciation objective de la situation de l'étranger retenu en zone d'attente. User d'un critère temporel pour limiter la possibilité de soulever certaines irrégularités restreint considérablement le champ d'analyse du juge judiciaire, appelé à se prononcer sur la régularité de la mesure privative de liberté. L'impunité de certaines irrégularités faciliterait les détentions arbitraires et empêcherait le juge des libertés d'exercer un contrôle entier et effectif sur la privation de liberté de l'étranger. Or l'effectivité du contrôle du juge est une condition primordiale de la régularité de l'atteinte à la liberté au sens de l'article 66 de la Constitution comme de l'article 5 de la CEDH.

Une telle limitation est donc contraire à la fois à l'article 66 de la Constitution et à l'exigence constitutionnelle reconnaissant le droit à un recours effectif et à un procès équitable, d'autant plus que cet article 12 est susceptible de s'appliquer aux cas envisagés aux articles 10 et 11 sus-analysés. Nous demandons donc au Conseil de juger l'article 12 de cette loi contraire à la Constitution, en son article 66, et à la Déclaration des Droits de l'homme et du citoyen, en son article 16.

\section{B. L'étendue du pouvoir du juge et l'effectivité de son contrôle}

Article 13: «L'existence de garanties de représentation de l'étranger n'est pas à elle seule susceptible de justifier le refus de prolongation de son maintien en zone d'attente».

L'article 66 de la Constitution n'autorise certaines atteintes à la liberté individuelle qu'à la condition de les soumettre au contrôle du juge, et qu'elles respectent l'exigence de nécessité et de proportionnalité quant aux objectifs poursuivis. L'État doit trouver un juste équilibre entre l'intérêt général et les droits individuels. Ces mesures privatives de liberté ne sauraient être justifiées que si la préservation de l'ordre public en dépend.

L'article 13 prévoit que les garanties de représentation dont pourraient bénéficier les étrangers ne sont plus, à elles seules, susceptibles de justifier un refus de prolongation de la mesure privative de liberté.

Ces garanties de représentation consistent notamment en la possession par l'étranger d'un billet de retour vers son pays d'origine, d'une réservation hôtelière, d'une somme d'argent en espèces, ou encore de la présence en France d'un membre de la famille. À elles seules, elles étaient jusque-là considérées comme les preuves d'un faible risque à laisser entrer la personne sur le territoire.

Dès lors, elles constituent un élément essentiel que le juge doit prendre en compte lorsqu'il apprécie la nécessité de prolonger la mesure privative de liberté. En interdisant au juge de se fonder uniquement sur l'existence de ces garanties de représentation pour refuser la prolongation du maintien en zone d'attente, l'article 13 restreint considérablement les pouvoirs du juge dans le contrôle qu'il exerce sur la nécessité du maintien de l'étranger en zone d'attente. Cet article remet en cause non seulement la proportionnalité et la nécessité des atteintes à la liberté individuelle, le rôle central joué par ces garanties, mais aussi l'effectivité du contrôle du juge exigée pourtant par l'article 66 de la Constitution.

Nous vous demandons donc de censurer l'article 13 de cette loi comme contraire à la Constitution, en son article 66, et à l'article 16 de la Déclaration des Droits de l'homme et du citoyen consacrant le droit à un recours effectif. 